\title{
Evaluational Brainstorming
}

\author{
Claudio Bezzi \\ Consultant Evaluator, Perugia, Italy \\ Email: bezzi@valutazione.it \\ Received May 16 ${ }^{\text {th }}$, 2011; revised July 24 $4^{\text {th }}, 2011$; accepted September $7^{\text {th }}, 2011$.
}

\begin{abstract}
Among the evaluation techniques based upon group queries (e.g. focus group), brainstorming does not enjoy particular consideration. This might be the result of its origin and development within organizational and managerial domains, traditionally focused more on "idea production" (and problem solving) than on idea analysis within the context of evaluational and social research. This paper presents a development of classical brainstorming, which is quite useful to evaluation, where the traditional idea-producing step is followed by group analysis and exploration of the shared evaluand-specific semantic space. This evaluational brainstorming is the result of a shared understanding of the evaluand by different stakeholders, who can now ascertain their goals and draw cognitive maps to guide subsequent methodological choices and data gathering requirements.
\end{abstract}

Keywords: Brainstorming, Participate Evaluation, Social Research, Research Techniques

\section{Introduction}

Brainstorming has yet to become one of the common techniques of evaluators and social researchers alike because of its significant identification with business consultants, and its lack of operative impact. Brainstorming is an exploratory technique, open to new meanings, proposals and ideas, but it seems that it does not provide operative synthesis, useful to evaluational research.

Nevertheless, once innovatively upgraded, brainstorming can become a very powerful tool, allowing:

- The evaluand's goal clarification.

- A so-called evaluand's semantic exploration, in order to define the correct evaluational path and choose the best evaluation strategies.

- An agreement, among the different stakeholders participating in the evaluation, on shared meanings, which constitutes the crucial element needed to share processes and outcomes.

In this article I plan to describe the "evaluational brainstorming", i.e. a brainstorming tailored to support evaluational research. This methodological proposal has been successfully experimented upon in more than ten years of evaluation activities, on different themes, with groups of different nature and size, experimenting with different operational modes. This paper thus become a synthesis of the best solutions experimented on so far ${ }^{1}$.

In almost the same period in which Lazarsfeld and Merton

\footnotetext{
${ }^{1} \mathrm{~A}$ much more exhaustive version, including variants, specific cases and a robust theoretical apparatus supporting the technique, is available in Italian: Claudio Bezzi and Ilaria Baldini, Il brainstorming. Pratica e teoria, Franco Angeli, Milan, Italy 2006.

${ }^{2}$ Osborn, A. F. (1953). Applied imagination. Principles and procedures of creative thinking, New York, NY: Charles Scribner's Sons. Although brainstorming literature is quite vast, normally consists of operator's manuals, written for professional corporate consultants and managers, or superficial "ten-step" quick guides. A minimal bibliography, useful to further the study of classical brainstorming includes Rawlinson 1986; Cory and Slater 2003; Rich 2003; Streibel 2003. On electronic brainstorming, all legitimate doubts aside, see Larey et al. 1996; Kay 1994. In the 90's an animated debate took place on the alleged value of group interaction in brainstorming, contrasting the well known positive strength value usually attributed to it. On this subject, see Diehl and Stroebe (1987; 1991); Mullen, Johnson and Salas (1991); Stroebe and Diehl (1991; 1994); and Stroebe, Diehk and Abakoumkin (1992).
}

created the focus group at the Bureau of Applied Social Research, an American business consultant successfully started a movement to develop creativity within the manufacturing industries (in addition to arts and sciences), offering innovative analytical solutions to problem solving. One of these solutions, the so-called brainstorming, showed an unusual approach to groups and had autonomous fate and fame, distinct from the larger body of original proposals. The consultant was Alex Osborn, and in his major work, Applied Imagination, he dedicated to brainstorming only a few pages at the end of the last chapter ${ }^{2}$.

Since then, brainstorming has been implemented by a great number of managers and consultants, and studied by a few psychologists, without eliciting much interest among social researchers. In addition to its origin and non-academic milieu, brainstorming was seen as lacking a real research application. After all, this is a group management technique geared to generic "idea production". In fact, the term has been adopted by our common lexicon and associated to the free (and often chaotic) expression of one's thoughts. "To have a brainstorm with my peers" means "let's say what comes to mind about our problem, without formality or pretensions of expressing fully articulated thoughts from the start.” This approach is useful in starting design processes (research included), but does not convey information within a structured design of social analysis.

The undeniable revival of constructivist approaches in evaluational research, and the offerings of techniques based on a dialogic exchange within a group (e.g. focus groups) has renewed the interest on brainstorming as well. With a simple condition: beyond its function as a problem solving tool, brainstorming has to be shown to detect information within the framework of research methodology.

Such a result could be achieved introducing a new and improved version of this technique, which I call "evaluational brainstorming".

The evaluational brainstorming can find several applications, but its main feature is its suitability to explore a concept or an evaluand and build its possible conceptual map. Brainstorming final product is a set of indicators of the explored concept (similarly, in evaluation, a set of evaluand's indicators) built up by a group of experts, operators, and relevant stakeholders. It is 
a group process and, as such, tends to define a shared representtation of that concept or evaluand.

\section{How Does Evaluational Brainstorming Work?}

The proposed brainstorming is carried out in the following sequential stages:

- Creative phase (Osborn's classical brainstorming).

- Classification phase, an inductive process.

- Synthetic phase, a deductive process.

Space considerations force us to overlook important preliminary remarks on group selection and recruiting, the initial welcome of the participants, and the set and setting in which the brainstorming session should take place. By and large, the indications are well known, similar to those applied to likely techniques (such as the focus group) and dictated by a modicum of common sense. Follows a summary of brainstorming management, phase by phase.

\section{Creative Phase}

Firstly, the facilitator describes the rules of the creative phase of brainstorming as follows:

- Produce as many ideas as possible.

- Do not censor yourself.

- Do not censor others.

With minimal modifications, these are the rules set by Osborn himself, and have remained unchanged for decades. In reality, Osborn's fundamental idea is the separation between the moment of idea production and the critique and discussion of the ideas thus produced. In fact, the critique can bring the meeting to a halt, making it sterile with excessive critical fervor aimed to the ideas which are voiced first, to the detriment of other proposals to be explored — at a later time — in order to find the best solution to the problem at hand.

Subsequently, the facilitator explains that "no censorship" means avoiding both considering trite one's ideas, and devaluating the ideas of others, maintaining an open attitude towards different perceptions and interpretations.

Thus, the creative phase consists of an exercise in researching spontaneous ideas, concepts, and expressions pertaining to the focal issue. The facilitator can offer: "Well, now you can start saying everything that comes to mind on the brainstorming subject. What counts is the number of produced ideas and concept, therefore I would urge you to respect the no-censorship rules".

The facilitator immediately writes each idea expressed by the participants on a white board. If someone expresses an articulated thought to be retranslated in a few words, the facilitator should ask to restate and simplify it, reminding the group that s/he can write on the white board only single words, single concepts or short expressions, the so-called 'strings'.

From the linguistic standpoint, the strings are quite a diversified set of linguistic signs, which can include the following:

- Predicative sentences (or simpler still: sentences with a verb, which are grammatically correct and obvious, i.e. "Payments are always late"; "The staff is quite tired of it"; "We need to pay attention to the user"; etc.);

- Nominal sentences (without a verb, such as "Enough of it!” or "Slave drivers!");

- Various rhetorical figures, mainly: metaphors ("That's another can of worms"; "We are like sheep among wolves"; etc.), metonymies ("The Man never leaves the upper floor"; "We do not need brain, just a strong stomach"; etc.) and synecdoche ("The service works", to indicate staff, structures and services of a specific center or unit; "Our bread and butter"; etc.); allusions, antiphrases (e.g. "We have everything we need!”, meaning we lack everything), periphrases, and the vast rhetorical and stylistic spectrum, which is as diversified as the participants and as understandable as their culture and the overall context (when in doubt, and while s/he writes, the facilitator asks "what does it mean?");

- Deixis and other exophora (or "indexical” sentences, which can be understood only within their context, such as "Up to here [with an ostensive gesture]"; "They [the users] keep calling"; etc.);

- Single words (substantives, adjectives, verbs, inflected in various ways, which acquire a specific meaning according to their reference context, for example: "Honesty [a team quality]"; "Training [what is required]"; "Traveling [the result of a new policy]"; etc.).

This listing suffices to show the extreme heterogeneity of strings produced during a brainstorming (first phase). Generally, predicative sentences are rare, and it is hard to imagine a brainstorming that produce only predicates. The session would be stifled and boring, lacking the typical creative stage rhythm. But a brainstorming producing only words, as exemplified last (the most frequent type) would equally sound a bit too ritual, especially if is not interleaved with nominal sentences (in general, exclamations), metaphors and deixis. Usually, the individuals who are more creative, restless, expert, and mature (in other terms and in all probability, the leaders) tend to both break the order of predicates and simpler lexemes and to provoke the group, explicitly highlighting a concern or indicating a dimension by means of targeted emphasis, metaphors, metonymies, exophora, and stronger expressions.

Strings thus produced become semantic tiles, the basic units that describe the object of interest.

Overall, the creative phase has the following objectives: exploration; agreement on meanings; and interpretational expression of the object of interest (the central concept). This is achieved through an untidy and disorderly, albeit exhaustive analysis.

The facilitator can think in advance the main areas/dimensions featured by the central concept, on the basis of his/her mandate and brainstorming objectives.

Once this set-up is in place, the facilitator can concentrate his/her attention on some dimensions which are considered conceptually important. For example, if the research is focused on "elderly home care", areas such as "funding”, "organization" or "home care objective definition" cannot be forgotten or ignored. If the group participants do not address such issues, the facilitator has the power to discretely suggest them, perhaps at session's end or in a moment of rest and group disorientation.

\section{Classification Phase}

A this stage, "classic" brainstorming is over and the facilitator has to deal with a large amount of strings waiting to be analyzed. According to classic routines, the activities of analysis, selection and implementation of the "best" ideas are performed by the manager, a manager's designee, a small group, etc. Generally, the group that has generated those ideas is not involved, the reason being that in a business environment ideas need to be adapted to the firm's overall strategy, the company mission, or simply the CEO's whims.

String analysis is the divide bridged by our brainstorming. 
Starting with brainstorming second phase, the analysis is performed by the very group producing the ideas, according to completely different rules, which must be explained to the participants.

Because the goal of evaluational brainstorming is the inductive construction of an indicator set for the explored concept, the group needs to be guided toward specific cognitive activeties, in order to reflect on the possible groupings, by homogeneous classes, of the collected strings. Classification-brainstorming second phase-is implicitly associated to the concept dimensions, and must be achieved by inductive processes. Instead of establishing classes in order to assign strings to them, it is paramount to work on the strings, massing them together until the group judges that the emerging classes are coherent. It is a true work of meaning's construction, avoiding as much as possible the burden of past ideas about and upon the concept. The group is focused on the individual strings (see below) and mediates their semantic and pragmatic values by pairing them more and more in larger clades, which only afterward show their inner coherence as classes (dimensions) both homogeneous and characterized.

When the first phase is considered completed, the facilitator invites the group to review its output and, starting with the first string, suggests: "Now, our task is to assemble or put together strings that appear to have some elements in common, no matter the reason. To this effect, if this is the first string, I'll read those following and you'll tell me which ones have something in common with the first. I'll place a marker (e.g.: a red triangle) next to the strings you select as similar or coherent to the first. Then we'll repeat the process with another string, using a different marker (for instance, a blue square), and we'll continue until we will analyze all of them."

This task presents the following facets:

- It requires the creation of homogeneous groups (also classes, sets, categories or families, as long as the participants understand the concept), containing strings linked by semantic affinities. Hence the "classification" phase label.

- If conceptually more abstract, a string can be marked by several symbols, because it shares diverse elements with many other strings, thus belonging to more than one class.

- When they are too abstract, strings can produce the socalled "omnibus" classes, containing a large quantity of broad-spectrum strings that are both generic and somewhat useless in terms of further processing. If the facilitator suspects such a classification, s/he should express his/her concern, guiding the analysis toward other classification criteria.

- Classes should have as much as possible a limited semantic extension, without assuming too abstract a feature;

- The classification phase is based upon an additive method, because it requires the analysis of one string at a time, judging whether it can be associated to the reference string. In this way the group does not immediately perceive the emerging classes, but simply defines the logical nexus linking a string to others.

As a result, different classes are formed, each marked by a different symbol.

These classes can be immediately named. This is much easier when the meaning of each class is finite and the participants recognize by themselves that they are dealing with a class as such. In some cases, though, a strong connotation is absent (e.g.: because the class is too abstract, see above.)

Nevertheless, to conceptually define the meaning of these classes is a task reserved to the third, synthetic phase. In the current phase, the group discussion may focus on whether it is appropriate to include a given string in one class or another. This approach encourages the expression of the links between concepts (or strings). By the same token, because in this second phase the group builds reality representation criteria (thru classification), it is quite likely that-at some point-the group expresses diverging visions. In reality, whether to include or not a string in a given class is not a purely logical operation, but impinges upon the string interpretation offered by each individual actor. Thus, this interpretation shares to a fair degree the semantic plane (pertaining to words' meaning), and is quite convergent to the pragmatic level (pertaining to words' usage).

Obviously, the first phase rules (do not censor, do not criticize) do not apply any longer. Even better, it is inevitable and often useful, to have a discussion clarifying and mediating the shared framework of meanings.

\section{Synthetic Phase}

The third phase is dedicated to indicators.

It is preceded by a break during which the participants can relax and the facilitator can transcribe all of the strings on separate sheets, one for the strings with the red triangle (which by now could have been named by the group), another for the strings with the blue square, etc.

The group will work class by class (or dimension by dimension), without the confusing interference of page after page of strings decked with different symbols, and will have the opportunity to observe the whole picture and-deductively—catching its essential elements that lead to the indicators.

The facilitator starts: "Please note that the strings are organized according to the previous classification. Now we should revisit the strings order to find further subgroups within them. Observe the first sheet and tell me if you can find new string groupings within this class, based on their common elements”.

At this point, the following can happen:

- No further groupings emerge within a class. This happens when the class contains a few strings very coherent and semantically close. In this case, the participants can only attribute a name to the class, if it doesn't have one already;

- Several groupings emerge within a class. These groups clearly refer to elements, processes, objects, concepts that can be differentiated even within a class already defined as homogeneous. In this case, the facilitator marks with the same symbol, (e.g., a number) the strings that cam be grouped within that class, asking the participants to name the sub-class thus formed. The work continues for all strings within the class.

- The third phase goals can be summarized as follows::

- Recognition of the classes built during phase 2.

- Definition of sub-classes within said classes and their denomination.

- Attribution of meaning to the work done so far, ordering what was disorganized.

The final result of the third phase is the individuation of indicators of the explored concept. In the same way in which classes were related to the concept dimensions, its specific elements are referred to indicators.

Generally speaking, in reality the group is never confronted with ideas such as concepts, dimensions and indicators. In fact, this approach generates inductive group thinking, linked to a communication pragmatics process, fostering the group's stipulation of reality's particular meaning within the context of the explored concept. 


\section{From Brainstorming Strings to Indicators}

This process is the net opposite of the logic-deductive operation performed by the researcher and his team while involved in their arm-chair conceptual exploration, the Top-Down process typical to Lazarsfeld's paradigm (Lazarsfeld 1958).

Figure 1 shows a simplified version of Lazarsfeld's paradigm, illustrating the role of strings and the differences between the two approaches. As shown in this figure, Lazarsfeld's paradigm (which is basically rationalistic, typically adopted by researches, including those concerned with evaluation) is a deductive process that explores the concept breaking down its dimensions, and eventually its sub-dimensions (from top to bottom in Figure 1). From these sub-dimensions, the indicators are finally chosen and further defined at the operative level (the empirical mode of information detection). On the contrary, our brainstorming is an inductive process. The search for indicators is guided by their logical underpinnings and culturally implicit factors, and it is by progressive accretion that the concept emerges equivalent to the research object and-in evaluation - the evaluand (from bottom to top in Figure 1).

To work inductively with a group is to search latent meanings, sedimentations of professional and corporate culture, idiomatic expressions. This is the material expressed by the strings that are generated during the first phase of brainstorming. They form the raw material from which the indicators are forged. According to inductive and group logics, the translation of strings into indicators requires the study of the concept dimensions, which takes place during the second phase.

Naturally, the basic approach is constructivist, and it pivots on the shared construction of a given reality. When brainstorming is used in evaluation, this framework is very useful, especially when the participants are also the evaluation future users. In this case, we have to deal with their corporate culture, the very way in which they work and represent reality (Bezzi 2006).

The following (clearly symbolic) elements of Figure 1 deserve attention:

- The strings are not the indicators' further sub-specification (as the case concept/dimensions/indicators), but autonomous linguistic expressions. In most cases, they are linked to specific indicators (as shown by the dashed arrows connecting strings and indicators). It is also easily seen (both in Figure 1 and brainstorming sessions) that some strings are directly referred to dimensions or, more rarely, the very concept. Our goal is to extract indicators from the collected strings, but each string is per se neither a ready-made indicator nor one of its parts.

- Strings can characterize several dimensions and indicators at once, exactly as each indicator can refer to more than one dimension or concept at a time.

- It is possible to end with oddly placed strings. These strings appear to refer to dimensions the group cannot isolate and identify (see the far lower right string, referred to an unknown and dashed indicator). Last but not least: the sign and pragmatic context in which the strings are somewhat freely placed in this or that class is neither arbitrary nor random. In reality it should be viewed as the analogy to the Lazarsfeld's principle of index interchangeability:

Each indicator has a specific character and should never be considered as fully representative of the searched for classification (Lazarsfeld, 1958)

Similarly and a fortiori, due to their proto-indicator nature, strings represent categories only in an approximate, circumstantial and, more importantly, contextual fashion.

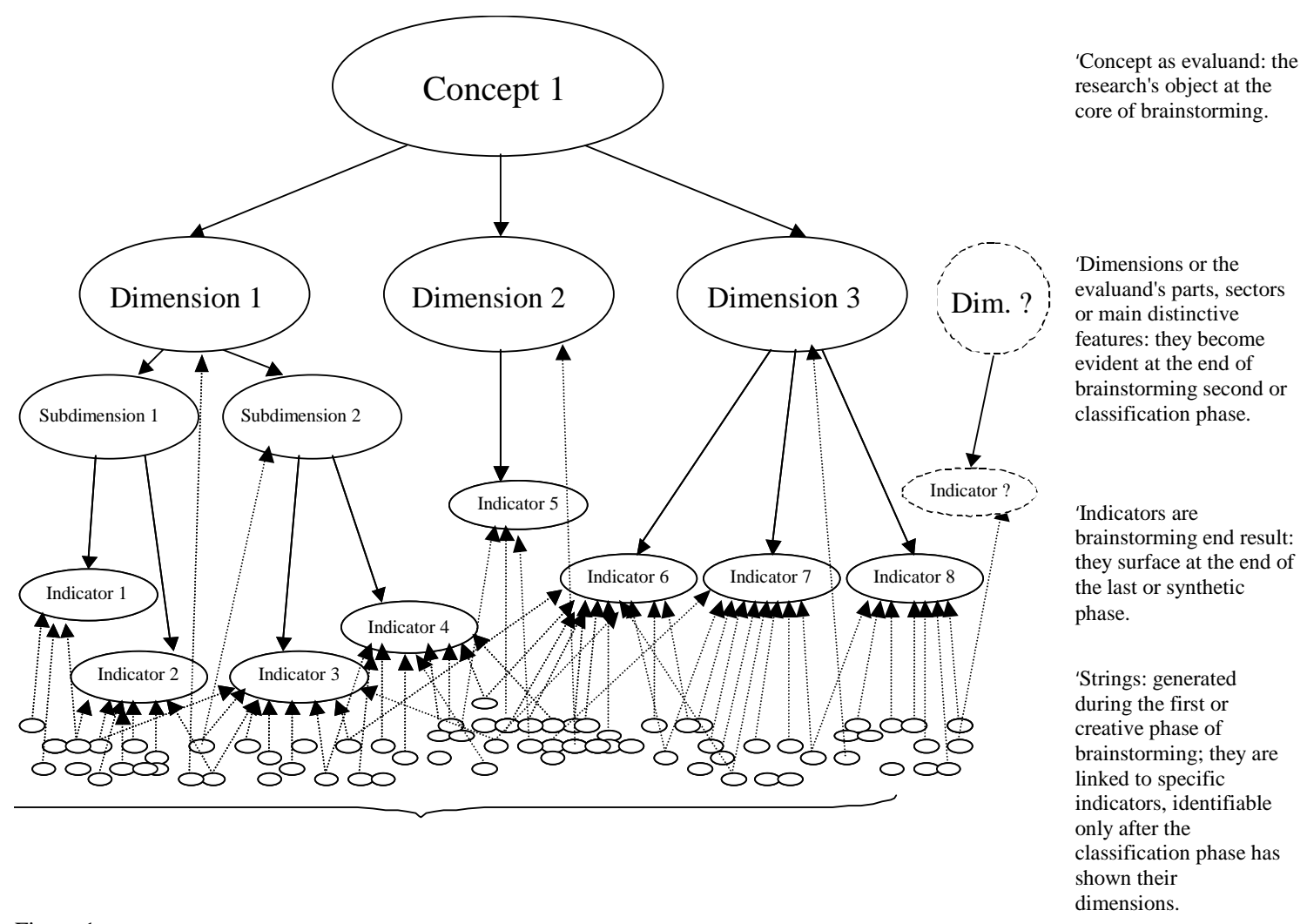

Figure 1.

Lazarsfeld's paradigm and brainstorming logic. 


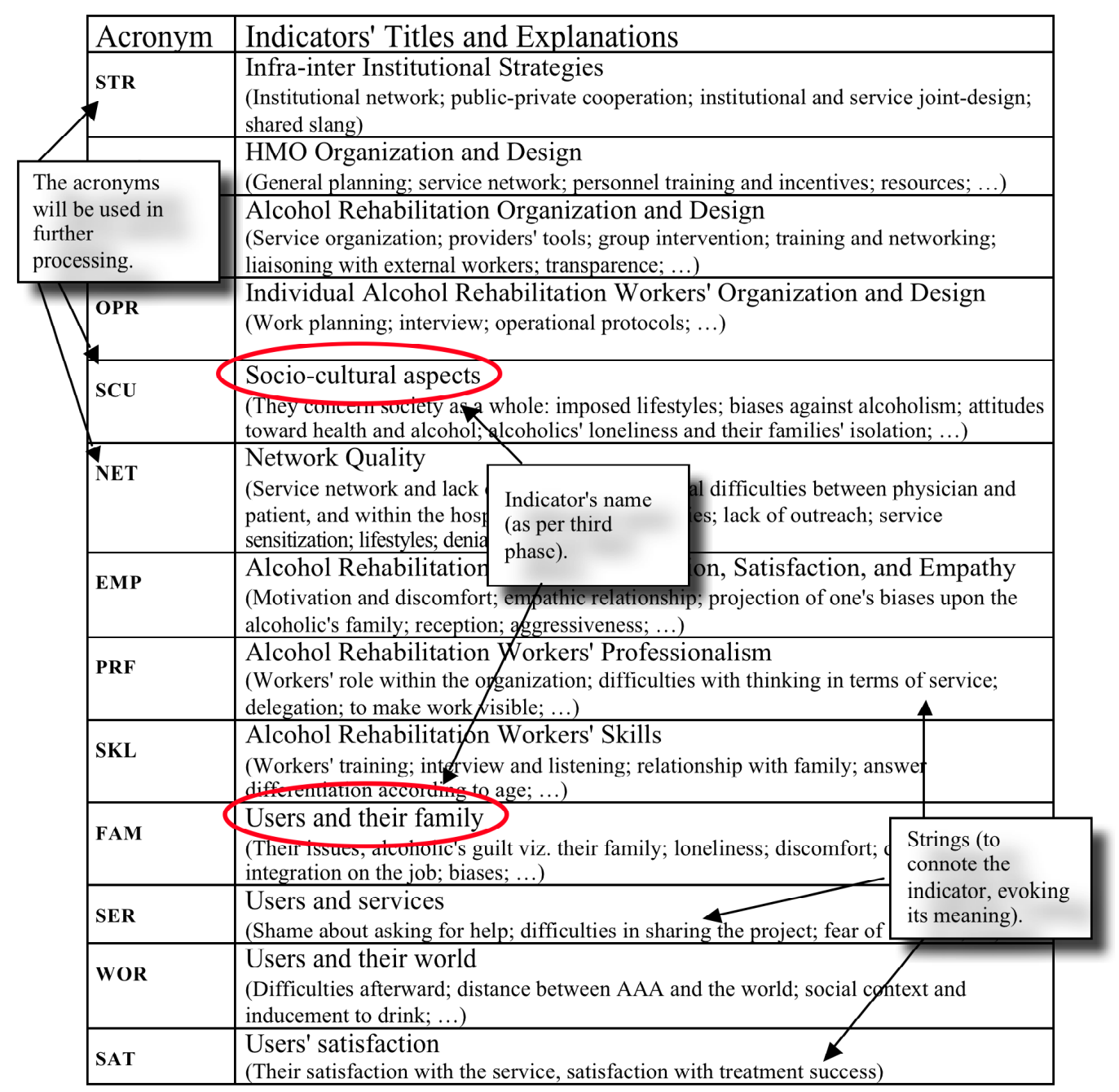

Figure 2.

(Partial) example of evaluational brainstorming rendering about an alcohol rehabilitation service.

- In any case, brainstorming rendering, and its subsequent research uses (from the transformation of indicators into questions of a survey to conceptual mapping to be used in evaluation) must preserve as much as possible the pragmatic indicators' substrate built up during phase 3 . To this effect, each indicator, in addition to its group-selected denomination, is shown with its string set, because of its high evocative value, especially felt by the very group that generated them (Figure 2 shows an example of evaluational brainstorming rendering.).

\section{References}

Bezzi, C. (2006). Evaluation Pragmatics. Evaluation, 12, 56-76,

Bezzi, C., \& Baldini, I. (2006). Il brainstorming. Pratica e teoria. Milan, Italy: Franco Angeli,.

Cory, T., \& Slater T. (2003). Brainstorming. Techniques for new ideas. Lincoln, NE: iUniverse Inc.

Diehl, M., \& Stroebe, W. (1987). Productivity loss in brainstorming groups: Toward the solution of a riddle. Journal of Personality and Social Psychology, 53, 497-509. doi:10.1037/0022-3514.53.3.497

Diehl, M., \& Stroebe, W. (1991). Productivity loss in idea-generating groups: Tracking down the blocking effect. Journal of Personality and Social Psychology, 61, 392-403.

doi:10.1037/0022-3514.61.3.392

Kay, G. (1994). Effective meetings through electronic brainstorming.
Journal of Management Development, 14, 4-25.

Larey, T. S., Leggett, K. L., Paulus, P. B., Putman, V. L., \& Evelyn, J. R. (1996). Social influence processes in computer brainstorming. Basic and Applied Social Psychology, 18, 3-14.

Lazarsfeld, P. F. (1958). Evidence and inference in social research, Dedalus, 87, 99-109.

Mullen, B., Johnson, C. \& Salas, E. (1991). Productivity loss in brainstorming: a meta-analytic integration, Basic and Applied Social Psychology, 12, 3-23. doi:10.1207/s15324834basp1201 1

Rawlinson, J. G. (1986). Creative thinking and brainstorming, Aldershot: Gower Publishing.

Rich, J. R. (2003). Brain storm. Tap into your creativity to generate awesome ideas and remarkable results. Franklin Lakes, NJ: Career Press.

Streibel, B. J. (2003). The manager's guide to effective meetings. New York, NY: McGraw-Hill.

Stroebe, W., \& Diehl, M. (1991). You can't beat good experiments with correlation evidence: Mullen, Johnson, and Sala'S meta-analytic misinterpretations. Basic and Applied Social Psychology, 12, 25-32. doi:10.1207/s15324834basp1201_2

Stroebe, W. \& Diehl, M. (1994). Why groups are less effective than their members: on productivity losses in idea-generating groups, European Review of Social Psychology, 5, 271-303. doi:10.1080/14792779543000084

Stroebe, W., Diehl, M., \& Abakoumkin, G. (1992). The illusion of group effectivity, Personality and Social Psychology Bulletin, 18, 643-650. doi:10.1177/0146167292185015 